\title{
Layered Montmorillonite Mineral Clay Used for Controlled Insulin Release
}

\author{
Sonmez Maria ${ }^{1}$, Ficai Denisa ${ }^{2}$, Ficai Anton ${ }^{2}$, Trusca Roxana ${ }^{3}$, Dana Florentina Gurau ${ }^{4}$ \\ ${ }^{1}$ Research Institute of the University of Bucharest \\ 36-46 Bd. M. Kogalniceanu, Bucharest, Romania \\ ficaimaria@yahoo.com \\ ${ }^{2}$ Politehnica University of Bucharest, Faculty of Applied Chemistry and Material Science \\ 1-7 Polizu St., Bucharest, Romania \\ denisaficai@yahoo.ro; anton.ficai@upb.ro; \\ ${ }^{3}$ S.C. METAV R\&D S.A \\ 31 C.A. Rosetti str., \\ Bucharest, Romania \\ truscaroxana@yahoo.com \\ ${ }^{4}$ National Research \& Development Institute for Textiles and Leather-division: Leather and Footwear Research \\ Institute \\ 93 Ion Minulescu St., Bucharest, Romania \\ dana.gurau@icpi.ro
}

\section{Extended Abstract}

Subcutaneous insulin injections are currently the standard treatment for people suffering from diabetes. However, subcutaneous administration of insulin fails to maintain a steady blood glucose level and often leads to hypoglycaemia[12]. Lately, special attention has been paid to finding a carrier in which the drug would be dispersed or integrated in order to control the release rate and to achieve a stable concentration of the drug over a long period of time. Such a class of materials that can accommodate therapeutic compounds between their layers is that of layered materials. A promising inorganic material as carrier is montmorillonite (MMT), belonging to the smectites class. Such layered inorganic materials are not only inoffensive, but also very stable in acidic and alkaline media[3].

The purpose of this paper is to evaluate the release rate of insulin from the particles based on layered mineral clay (MMT) unmodified or chemically modified with functional groups such as aminopropyltriethoxysilane and octadecylamine (1.31PS Nanomer) or dimethyl dialkyl (C14-C18) amine (I.44P Nanomer).

Adsorption of insulin inside unmodified and modified MMT particles was carried out under vacuum using 70 IU of Humulin R human insulin dispersed in $3 \mathrm{ml}$ of distilled water relative to $1 \mathrm{~g}$ of powder MMT. After insulin adsorption, the powders were dried in a hot air oven at $40^{\circ} \mathrm{C}$ for 24 hours. The insulin powders were subsequently used in testing insulin release ability. In this regard, $0.30 \mathrm{mg}$ of each powder was weighed, after having been immersed in $20 \mathrm{ml}$ of phosphate buffer solution (PBS) with $\mathrm{pH} 7.4$ and at a temperature of $37^{\circ} \mathrm{C}$. Powders immersed in buffer solution were conditioned at $37^{\circ} \mathrm{C}$ and stirred continuously to best simulate existing conditions in the body. The calibration curve was made using a stock solution (PBS + insulin) with concentration of $0.14 \mathrm{IU} / \mathrm{L}$. This stock solution was further diluted with a set amount of PBS, in order to obtain standard solutions with concentrations ranging from 0.028-0.07 IU/L.

At a well-established interval, MMT powders immersed in PBS were placed in a centrifuge, at the following parameters: rotation speed - 10,000 rpm and holding time - $5 \mathrm{~min}$. From the clear solution resulting after centrifugation, an amount of $3 \mathrm{ml}$ was extracted by means of an automatic syringe and was introduced into a quartz cuvette and subjected to readings on a UV-VIS spectrophotometer to determine the insulin release rate. Readings were made during 0.15 - 124 $\mathrm{h}$ and at a wavelength of $201 \mathrm{~nm}$. MMT powders onto which insulin was adsorbed before the release tests were tested in terms of morphology (SEM) and composition (FTIR). The results obtained by FTIR show the presence of insulin in all types of particles tested, which proves that the adsorption occurred. Moreover, the presence of insulin could also be visualized by SEM. Powders recovered after testing the release of insulin were also tested by FTIR. The maximum insulin release rate was achieved when using unfunctionalized MMT powder, followed by the powder functionalized with dimethyl dialkyl (C14-C18) amine. 
Keywords: drug delivery, montmorillonite, insuline, buffer solution, adsorbtion

\section{Acknowledgement}

"Research project funded through the ICUB Fellowships for Young Researchers Program, 2016 Competition Project financed by the Research Institute of the University of Bucharest (ICUB)".

\section{References}

[1] Y. Y. Luo, X. Y. Xiong, Y. T. Z. L. Li, Y. C. Gong and Y. P. Li, “A review of biodegradable polymeric systems for oral insulin delivery," Drug Deliv., vol. 23, no. 6, pp. 1882-1891, 2016.

[2] T. M. Yasmeen, Md. Zubair, S. Begum and T. Muneera, "Various Emerging Trends in Insulin Drug Delivery Systems," Journal of Pharmaceutical Research, vol. 5, no.5, pp. 294-308, 2015.

[3] B. D. Kevadiya, G. V. Joshi, H. A. Patel, P. G. Ingole, H. M. Mody and H. C. Bajaj, "Montmorillonite-alginate nanocomposites as a drug delivery system: Intercalation and in vitro release of vitamin B1 and vitamin B6," Journal of Biomaterials Applications, vol.25, pp. 161-177, 2010. 